\title{
Design and Modeling of a High Accuracy, Three Degree of Freedom MEMS Manipulator
}

\author{
Shyam Venugopal ${ }^{1}$, Lun-Chen $\mathrm{Hsu}^{2}$, Smitha Malalur-Nagaraja-Rao ${ }^{2}$, \\ B.P. Wang ${ }^{1}, \mathrm{Mu}^{\mathrm{Chiao}}{ }^{3}$ and J.-C. Chiao ${ }^{2}$ \\ ${ }^{1}$ Department of Mechanical Engineering, ${ }^{2}$ Department of Electrical Engineering, \\ University of Texas at Arlington, USA \\ ${ }^{3}$ Department of Mechanical Engineering, University of British Columbia, Canada
}

\begin{abstract}
A new design for a high accuracy, 3-degree of freedom (DOF) MEMS manipulator is proposed. The 3DOF robotic manipulator is to be used for biomedical applications such as cell probing, tissue sampling, neuron signal reading and drug delivery, in which high accuracy and repeatability of positioning is required. While sensing or imaging elements are not available in the integration with the manipulator to provide feedback for positioning, we investigated a calibration approach to minimize the positioning errors. In-plane and vertical MEMS thermal actuators are chosen to perform the required tasks. The modeling of the thermal actuators was first studied and the results match with experimental results. A calibration algorithm is implemented to allow the minimization of accumulated motion errors. The algorithm was successfully applied to the manipulator and results were obtained. A MATLAB script was written to simplify the calibration procedure. Problems faced in the design and potential solutions will be also discussed.
\end{abstract}

Keywords: Manipulators, MEMS, calibration techniques

\section{INTRODUCTION}

Utilization of MEMS devices for micro-scale object manipulation provides applications in tools for minimally invasive surgery [1], bio-object micromanipulation [2], microfabrication such as fabrication of micro-nozzles [3] and assembly of micro-optical components [4], three-dimensional manipulation of carbon nanotubes [5], terabit storage systems [6] and microelectrodes for deep brain stimulation and neuronal recording [7-8]. MEMS devices provide high-precision positioning and high resolution of actuation. It makes the micro and nano-scale manipulation possible. The fabrication techniques of MEMS utilize batch processes that provide cost-effective mass production advantages. We proposed a probe-type manipulator consisting of a MEMS actuator chip in a minimally invasive surgical tubing for in vivo microscale object manipulation. The probe dimensions limit the inclusion of an imaging camera or a position sensor. Therefore, using visual or electrical feedbacks to provide close-loop control of the actuators while the probe in vivo becomes impossible. In this work, we investigated a calibration mechanism for the openloop manipulator in order to minimize positioning errors.

A MEMS robotic manipulator chip was designed to be mounted at the end of a tubing probe with a diameter of $1 \mathrm{~mm}$. The tubing could be flexible to allow surgical maneuver. The rigid head at the end of the tubing has a length of $8 \mathrm{~mm}$. The manipulator is to be the "end effector" of the probe and is required to have accurate positioning. Two of these manipulator chips are to be mounted at the end of the probe and used in tandem. Fig. 1 is a diagram of how the entire system looks like. Two identical MEMS chips are surfacemounted on either side of the central-axis divider.

Each manipulator is required to have three degrees of freedom (DOF) control. It is required to move up and down along the z-axis (linear/prismatic), back and forth along the $\mathrm{x}$-axis (linear/prismatic) and rotate about the z-axis (rotation). A manipulator with these three degrees of freedom is called to have a LLR or a PPR configuration. LLR manipulators are relatively accurate and easy to control.

Device and Process Technologies for Microelectronics, MEMS, and Photonics IV, edited by Jung-Chih Chiao, Andrew S. Dzurak, Chennupati Jagadish, David V. Thiel, Proc. of SPIE Vol. 6037, 60371Y, (2005) · 0277-786X/05/\$15 - doi: 10.1117/12.639904 


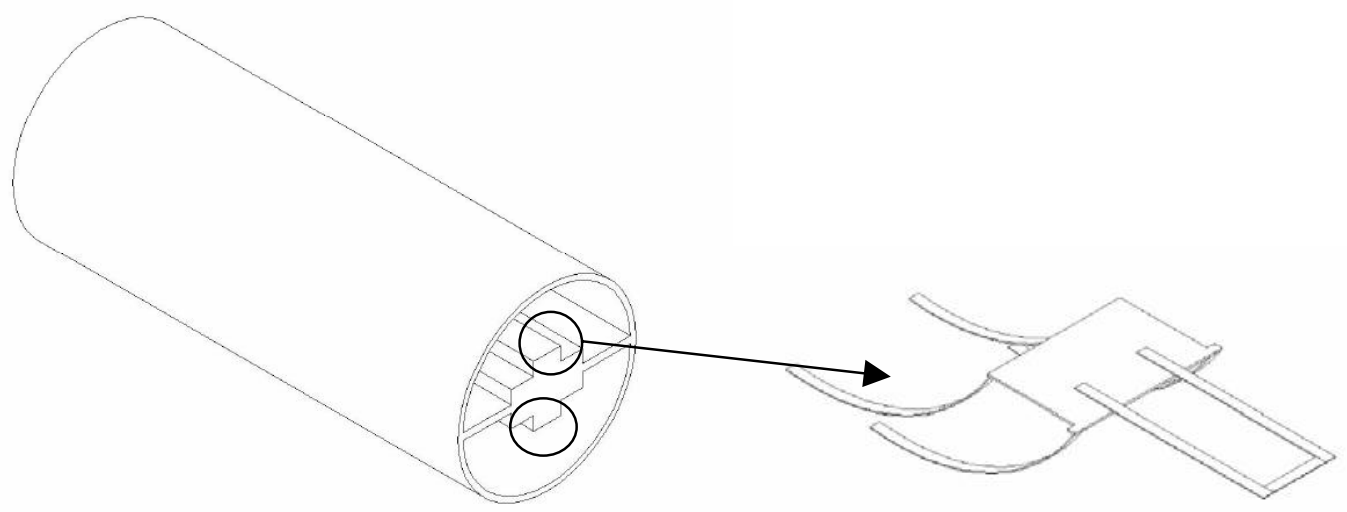

Figure 1: Probe configuration. The MEMS chip consists of actuators capable of providing a 3- DOF manipulation. Four vertical and two horizontal (lateral) actuators are used to provide the positioning.

\section{SYSTEM DESIGN}

\subsection{The MEMS Manipulator}

Both horizontal and vertical thermal actuators are used in the actuator design. Four vertical actuators are employed to allow the out-of-plane motion to lift a platform off the substrate. Two horizontal thermal actuators on the platform are to move the end effector (such as a microgripper [9-10] or microsurgical tools [11-12]). The horizontal thermal actuators are placed in a way to allow the end effector to rotate about the vertical axis. The configuration is shown in the Fig. 1

\subsection{Thermal Actuators}

A typical pseudo-morph thermal actuator has two arms - a hot arm and a cold arm. A displacement at the tip is generated due to the difference in the coefficients of thermal expansion. Thermal actuators can generate a relatively large amount of force for a low actuating voltage. The thermal actuator used in our design has two hot arms, which is based on the design by Burans et al [13]. The advantage of using two hot arms is to increase the force outputs of the actuator by a factor of 2 . The modeling methodology of such thermal actuators using the lumped-element approach based on the works done by Yan et al [14] was

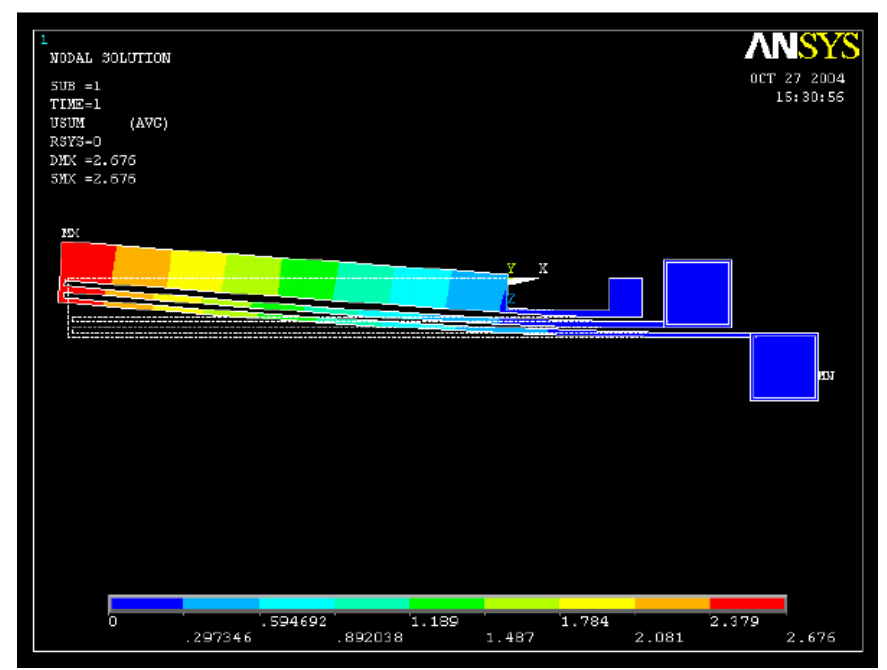

Figure 2: ANSYS simulation of the horizontal thermal actuator. 


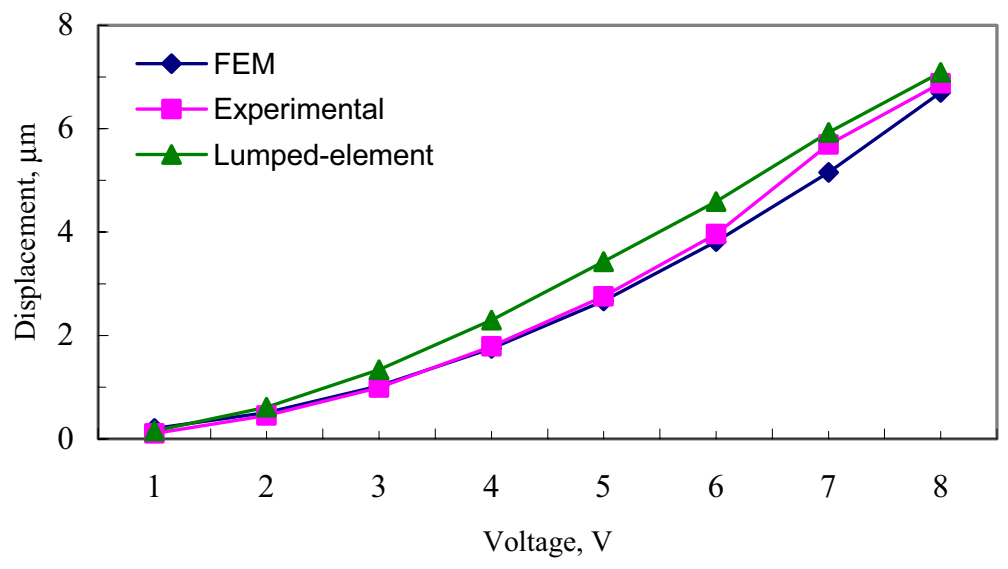

Figure 3: Comparison of displacements for the horizontal thermal actuator.

implemented with the Mathlab.

A finite-element model of the thermal actuator was also established using the ANSYS and the results were extracted from the models. A coupled field element Scalar Tet 98 was used in the meshing of the model.

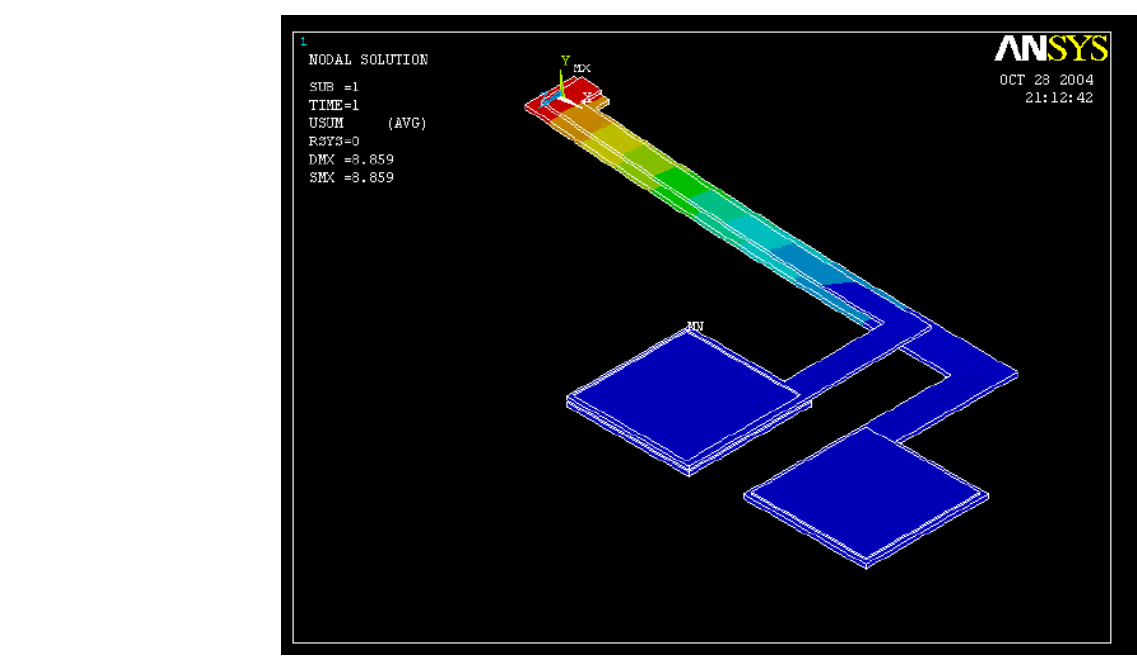

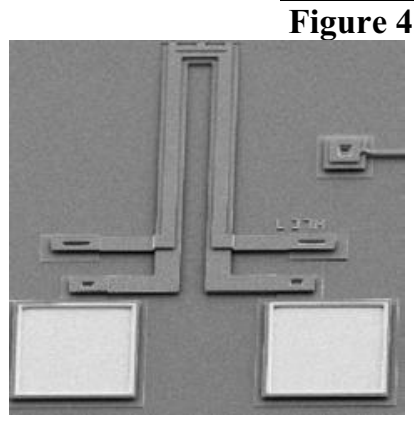

(a)

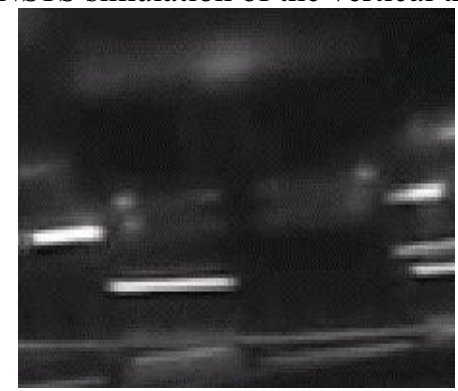

(b)

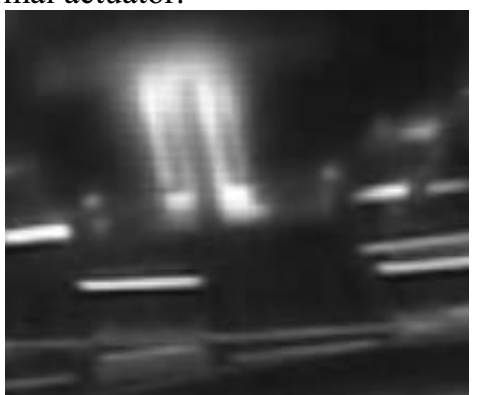

(c)

Figure 5: (a) An SEM photo of the vertical actuator. Video captures of the actuator (b) down and (c) up. 


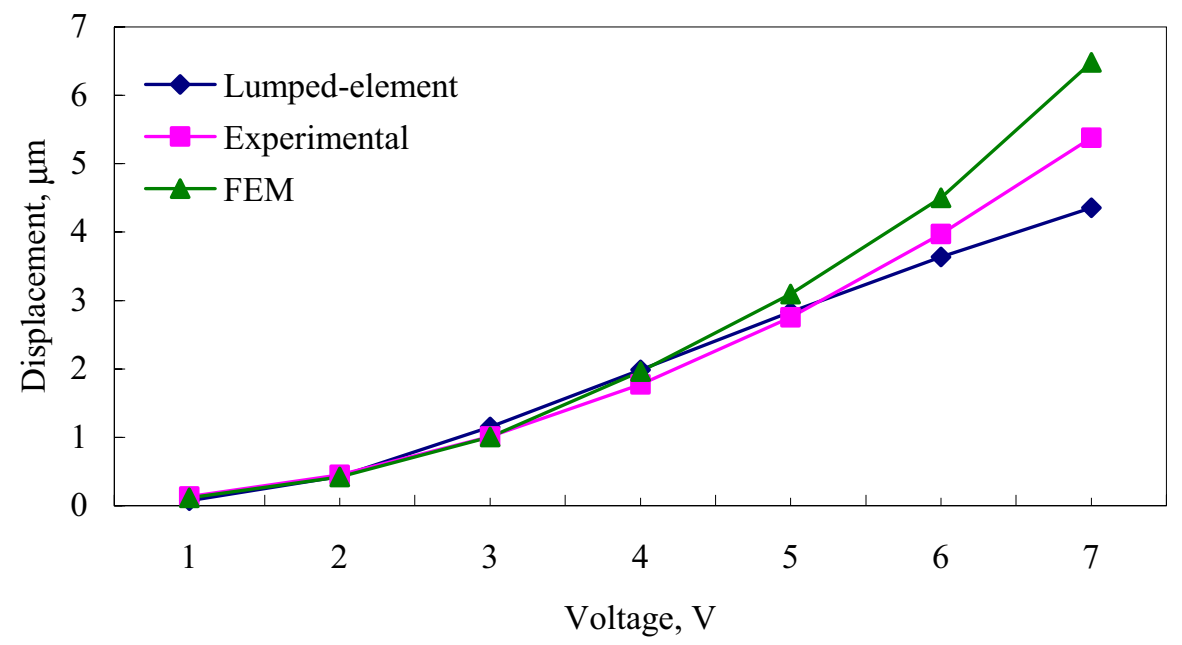

Figure 6. Comparison of displacements for the vertical thermal actuator.

Fig. 2 shows a typical simulation result with a certain biasing voltage. In our design, the polysilicon thermal actuators have a cold arm width of $14 \mu \mathrm{m}$, a hot arm width of $2 \mu \mathrm{m}$, and a gap between arms of $2 \mu \mathrm{m}$. The hot arms have lengths of $250 \mu \mathrm{m}$ and $220 \mu \mathrm{m}$ while the cold arm length is $160 \mu \mathrm{m}$. The applied voltage varies from 1 to $8 \mathrm{~V}$. Fig. 3 shows the comparison of simulation results using the lumped-element approach and the finite-element method (FEM) compared with the experimental data in [14]. The simulation results match with the previous works [14] within some discrepancy, possibly due to the excessive heat loss effects [15].

A vertical thermal actuator was designed with two layers of arms vertically stacked. The difference of thermal expansion in the arms makes the actuator moves up. With proper voltage applied, the actuator can also move down, on the top-layer arms if the residual stress causes the arms curl up at the room temperature. The design is based on the work done by Yan et al [16] as a bi-directional vertical actuator. The FEM analysis is similar to that for the in-plane thermal actuator. Since the model is symmetric, only half of the model is shown and simulated (Fig. 4). The same element Scalar Tet 98 is used in the meshing of the solid volume. The polysilicon actuators have a lower arm width of $18 \mu \mathrm{m}$, an upper arm width of $10 \mu \mathrm{m}$, and a gap between arms of $20 \mu \mathrm{m}$. The upper arms have a length of $170 \mu \mathrm{m}$ while the lower arm length is $210 \mu \mathrm{m}$. The applied voltage varies from 1 to $7 \mathrm{~V}$. Fig. 5 (a) shows a scanning electron microscope (SEM) photo of a vertical thermal actuator. Fig. 5(b) and (c) show video captured photos of the actuator when it is down and up, respectively.

The FEM model predicts a vertical displacement in one direction of $6.5 \mu \mathrm{m}$ for an input voltage of $7 \mathrm{~V}$ while the experimental data shows a 5.4- $\mu \mathrm{m}$ displacement. The results are shown in Fig. 6. The discrepancy in the FEM and lumped-element simulation may be due to the nonlinear behaviors and the excessive heat losses.

The two types of thermal actuators are used to provide the three degrees of freedom motion, PPR, of the manipulator. The vertical thermal actuators allow an up-and-down motion while the two in-plane thermal actuators provide the two remaining degrees of freedom. Controlling the difference in actuation between the two in-plane actuators can provide the rotation movement.

\subsection{Manipulator Modeling}

The Denavit-Hartenberg method is used to determine the forward and inverse kinematics of the manipulator [17] with three degrees of freedom motion in the prismatic-prismatic-rotary (PPR) fashion. 


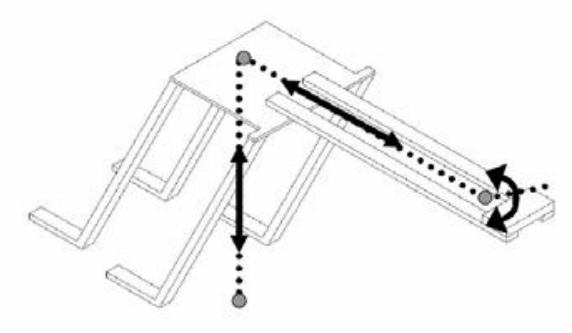

Figure 7: Equivalent kinematic diagram of the manipulator.

The forward kinematics deals with the issue of determining the position and orientation of the end effector, given a set of joint variables such as angles in the case of rotary joints and link extensions in the case of prismatic joints, as shown in Fig. 7. The DH parameters are used to create a transformation matrix (A $i)$ for the forward kinematics

$$
\begin{aligned}
A_{i} & =R_{z i, \theta i} \text { Trans }_{z i, \theta i} \text { Trans }_{x i, \alpha i} R_{x i, \alpha i} \\
& =\left[\begin{array}{cccc}
C \theta_{i} & -S \theta_{i} & 0 & 0 \\
S \theta_{i} & C \theta_{i} & 0 & 0 \\
0 & 0 & 1 & 0 \\
0 & 0 & 0 & 1
\end{array}\right]\left[\begin{array}{cccc}
1 & 0 & 0 & 0 \\
0 & 1 & 0 & 0 \\
0 & 0 & 1 & d_{i} \\
0 & 0 & 0 & 1
\end{array}\right]\left[\begin{array}{cccc}
1 & 0 & 0 & a_{i} \\
0 & 1 & 0 & 0 \\
0 & 0 & 1 & 0 \\
0 & 0 & 0 & 1
\end{array}\right]\left[\begin{array}{cccc}
1 & 0 & 0 & 0 \\
0 & C \alpha_{i} & -S \alpha_{i} & 0 \\
0 & S \alpha_{i} & C \alpha_{i} & 0 \\
0 & 0 & 0 & 1
\end{array}\right] \\
& =\left[\begin{array}{cccc}
\mathrm{C} \theta_{\mathrm{i}} & -\mathrm{S} \theta_{\mathrm{i}} \mathrm{C} \alpha_{\mathrm{i}} & \mathrm{S} \theta_{\mathrm{i}} \mathrm{S} \alpha_{\mathrm{i}} & \mathrm{a}_{\mathrm{i}} \mathrm{C} \theta_{\mathrm{i}} \\
\mathrm{S} \theta_{\mathrm{i}} & \mathrm{C} \theta_{\mathrm{i}} \mathrm{C} \alpha_{\mathrm{i}} & -\mathrm{C} \theta_{\mathrm{i}} \mathrm{S} \alpha_{\mathrm{i}} & \mathrm{a}_{\mathrm{i}} \mathrm{S} \theta_{\mathrm{i}} \\
0 & \mathrm{~S} \alpha_{\mathrm{i}} & \mathrm{C} \alpha_{\mathrm{i}} & \mathrm{d}_{\mathrm{i}} \\
0 & 0 & 0 & 1
\end{array}\right]
\end{aligned}
$$

where $C \theta_{i}=\cos \left(\theta_{i}\right)$ and $S \theta_{i}=\sin \left(\theta_{i}\right)$ and the DH parameters are listed in Table.1.

\begin{tabular}{|c|c|c|c|c|}
\hline Joint & $\theta_{\mathrm{x}}$ & $\mathrm{d}_{\mathrm{z}}$ & $\mathrm{a}_{\mathrm{x}}$ & $\alpha_{\mathrm{x}}$ \\
\hline 1 & $\mathrm{~d}_{1}$ & -90 & -90 & 0 \\
\hline 2 & $\mathrm{~d}_{2}$ & 0 & -90 & 0 \\
\hline 3 & 0 & $\theta_{3}$ & 0 & $\mathrm{~d}_{13}$ \\
\hline
\end{tabular}

Table 1: DH parameters used in the kinematic analysis of the robot.

The inverse kinematic relationships are derived to determine the joint parameters required to place the tool at a given co-coordinate in space [18]. The solution can be defined by more than one set of input parameters. We combine the forward kinematics and inverse kinematics approaches and the iterations between them are used in the error correction algorithm to minimize the errors in positioning.

\subsection{Errors and Error Correction}


Errors may accumulate in the MEMS systems due to many reasons such as manufacturing defects, internal stresses, hysterisis, noise (both thermal and electrical) and vibration. When loaded, other factors such as stresses, strains and bending also play important roles in the accurate positioning of the end effector. The errors accumulated can be compensated for by an appropriate calibration method. A calibrated robot typically could have accuracy one or two orders of magnitude greater than that of an un-calibrated one, especially with a closed-loop feedback method.

The in vivo end effector design, however, is an open-loop system. There are no sensors that could be mounted on the structures to provide feedbacks to the controller. In some surgical procedures, separate in vivo imaging probes are used to provide visual feedbacks. Simulations and sequential experimental tests could also be used to determine the average errors and they could be used to calibrate the structures. The kinematic designs of robots assume ideal conditions and parameters in the systems. In practical cases, the accuracy is limited without calibration.

\section{MODEL BASED CALIBRATION}

The model-based approach more accurately represents the actual kinematics of the manipulator system because it uses established relationships between true and measured values of the system to make corrections to the theoretical kinematic model. This method introduces correction factors to the parameters with errors and attempts to minimize the errors. This calibration procedure could correct the deterministic errors associated with fabrication, materials and design.

Two main iterative steps in the calibration procedure are (1) calculation of errors in the DH parameters and (2) correction of the joint parameters. The mathematical details of the steps involved are outlined in [19] and the DH parameters, forward and inverse kinematics computation details are in [18]. First, we derive the final position vector of the end effector using the forward kinematics. The calibration matrix, C, is calculated from by taking the partial differential for each component of the position vector with respect to each DH parameter. We then choose three calibration points and calculate $\Delta \mathrm{X}=\mathrm{C} \times \Delta \Omega$ with

$\Delta \mathrm{X}=\left[\frac{\delta \mathrm{f}}{\delta \theta}, \frac{\delta \mathrm{f}}{\delta \alpha}, \frac{\delta \mathrm{f}}{\delta \mathrm{a}}, \frac{\delta \mathrm{f}}{\delta \mathrm{d}}\right]$

where $\mathrm{f}$ is the position vector, $\theta, \alpha, \mathrm{a}, \mathrm{d}$ are the four DH parameters and $\Delta \Omega=[\theta, \alpha, \mathrm{a}, \mathrm{d}]$ is the DH matrix. We choose $45^{\circ}, 90^{\circ}$ and $135^{\circ}$ positions of the end effector for calibration. The speed of convergence depends on the number of points chosen. More points give a more accurate solution and faster convergence. The error vector $\varepsilon$ is calculated as the difference with the measured values $\mathrm{M}$ and the $\Delta \Omega$ and is solved from $\varepsilon=\operatorname{CM} \Delta \Omega$. The new DH parameters are obtained as $\Omega^{\prime}=\Omega+\Delta \Omega$. The iterations continue until the solution satisfies the required error limits.

\section{RESULTS}

Several different positions are chosen to test the calibration algorithm. A MATLAB script is written to carry out the calibration. A random number generator is used to produce $10 \%$ errors in the actuation voltages for both the in-plane and out-of-plane thermal actuators. For example, if the desired voltage is $5 \mathrm{~V}$, the actual applied voltages could be within $5 \pm 0.5 \mathrm{~V}$. Then the FEM simulations are carried out to find the estimated positions. The theoretical values are compared with the desired ones to carry out the calibration procedure. Figure 8 shows the results for the calibration algorithm A position at $(\mathrm{x}, \mathrm{y}, \mathrm{z})=(43.3,224,7.1)$ was chosen for the demonstration. Fifty statistical runs were carried out. The system could be successfully calibrated to reduce the predictable errors, and to a certain extent, control the effects by random errors. After calibration, positioning errors are controlled within the original $10 \%$ ranges.

\section{CONCLUSIONS}




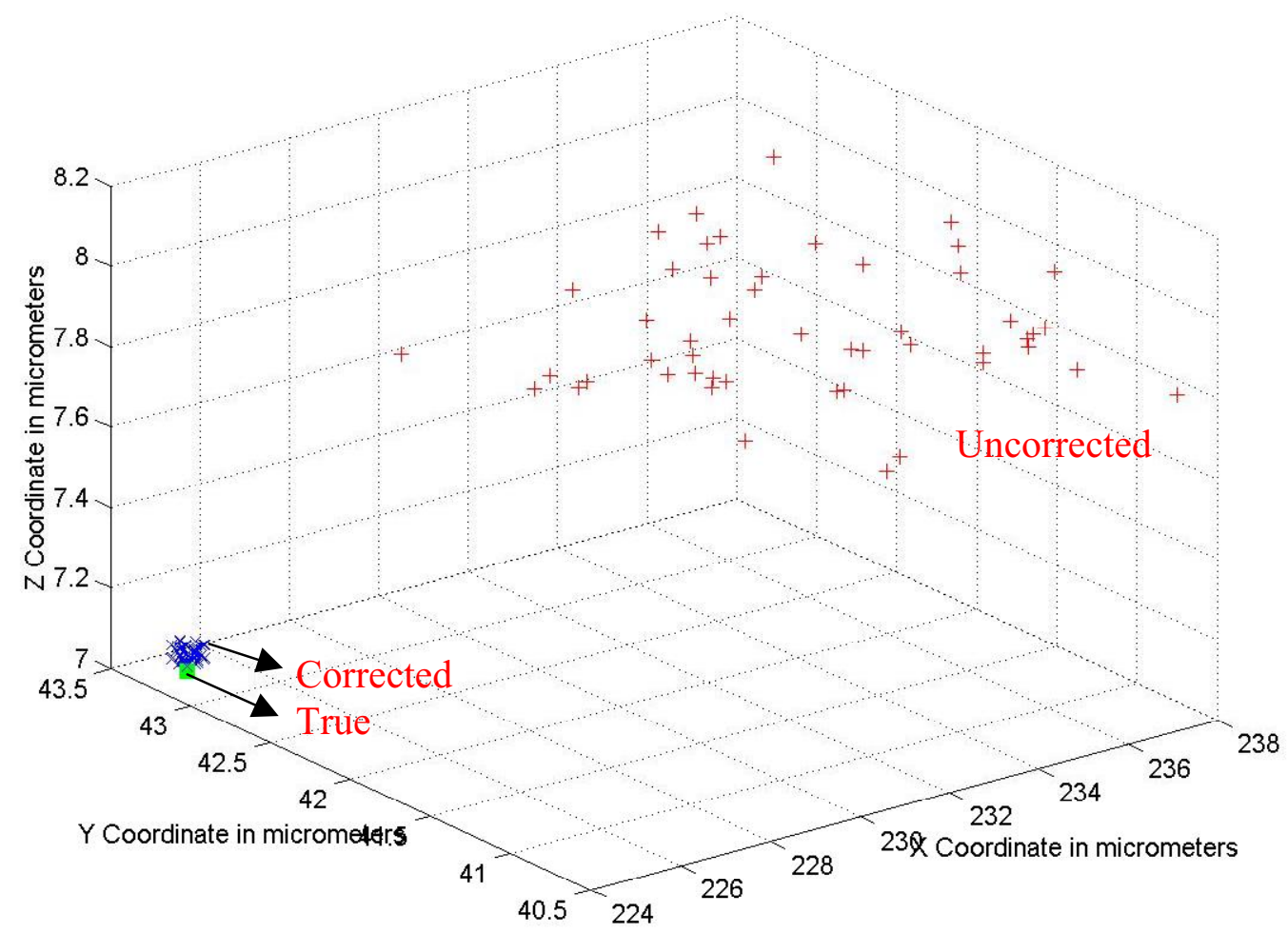

Figure 8: Results of the error correction algorithm. The "true" label indicates the desired position, the "uncorrected" points are the positions of the end effector before calibration with randomized errors in the actuators and the "corrected" points are the positions of the end effector after calibration.

A new design of a MEMS-based in vivo manipulator is presented. The designs utilize thermal actuators to provide the 3 degrees of freedom motion. FEM simulations are preformed to compare with the analytical lumped-element models and experimental results. A kinematic analysis is carried out on the structure of the manipulator and combined with the error correction algorithm for system calibration. Errors are introduced in the mathematical models and the algorithm is executed to show the capability of minimizing accumulative errors. The manipulator can be used in micro- or nano-manipulators to handle, probe, assemble or position 3-dimensional objects in micro-/nano-scales. The system feature also allows in vivo open-loop manipulation of small objects.

\section{REFERENCES}

[1] T. Kawai, K. Nishizawa, F. Tajima, K. Kan, M. Fujie, K. Takakura, S. Kobayashi, and T. Dohi "Development of Exchangeable Microforceps for a Micromanipulator System," Advanced Robotics 8, Vol. 15, No. 3, pp. 301-305, June, 2001.

[2] A. Kawaji, F. Arai, T. Sugiyama and T. Fukuda, "Three-dimensional Bio-micromanipulation," Advanced Robotics, Vol. 13, No. 3, VSP and RSJ, pp. 321-322, 1999.

[3] C.-L. Kuo, T. Masuzawa, and M. Fujino, "High-Precision Micronozzle Fabrication," IEEE Micro ElectroMechanical Systems '92, Travemunde Germany, pp. 116-121., Feb. 4-7, 1992.

[4] Lun-Chen Hsu, Varkey George, Dan Popa, Woo Ho Lee, Mohammed Mayyas, Ping Zhang, Harry E. Stephanou and J.-C. Chiao, "3D Microassembly Station," 2005 TexMEMS, The VII International Conference on Micro Electro Mechanical Systems, Sept. 21-22, 2005. 
[5] Min Feng Yu, Mark J Dyer, George D Skidmore, Henry W Rohrs, XueKun Lu, Kevin D Ausman, James R Von Ehr, and Rodney S Ruoff, "Three-Dimensional Manipulation of Carbon Nanotubes under a Scanning Electron Microscope," Nanotechnology 10, pp. 244-252, 1999.

[6] Edward Grochowski and Roger F. Hoyt, "Future Trends in Hard Disk Drives," IEEE Transactions on Magnetics, Vol. 32, No. 3, pp. 1850-1854, May 1996.

[7] T.H. Yoon, EJ Hwang, DY Shin, SI Park, SJ Oh, SC Jung, HC Shin, and SJ Kim. "A Micromachined Silicon Depth Probe for Multichannel Neural Recording," IEEE Transactions on Biomedical Engineering, Vol. 47, No. 8, pp. 1082-1087, Aug. 2000.

[8] Y. Hanein, K. F. Böhringer, R. C. Wyeth, and A. O. D. Willows, "Towards MEMS Probes for Intracellular Neuronal Recording," Sensors Update -- Sensor Technology - Applications - Markets, Vol. 10, No. 1, pp. 47-75, Wiley-VHC, 2002.

[9] N. Chronis, and L.P. Lee, "Polymer MEMS-based microgripper for single cell manipulation," 17th IEEE International Conference on Micro Electro Mechanical Systems, (MEMS), pp. 17-20, 2004.

[10] C.J. Kim, A.P. Pisano, R.S. Muller, "Silicon-processed overhanging microgripper," IEEE Journal of Microelectromechanical Systems, Volume 1, Issue 1, pp. 31-36, March 1992.

[11] Pak Kin Wong; U. Ulmanella, Chih-Ming Ho, "Fabrication process of microsurgical tools for singlecell trapping and intracytoplasmic injection," IEEE Journal of Microelectromechanical Systems, Volume 13, Issue 6, pp. 940 - 946, Dec. 2004.

[12] E. Perez-Arjona, M. Dujovny, H. Park, D. Kulyanov, A. Galaniuk, C. Agner, D. Michael, F.G. Diaz, "Stereolithography: Neurosurgical and medical implications," Neurological Research, Vol. 25, No. 3, pp. 227-236(10), 1 April 2003.

[13] D.M. Burans, VM Bright, "Design and Performance of a Double Hot Arm Polysilicon Thermal Actuator," Proc. SPIE, Micromachined Devices and Components III, Kevin Chau; Patrick J. French; Eds. Vol. 3224, pp. 296-306, Sept. 1997.

[14] Dong Yan, Amir Khajepour and Raafat Mansour, "Modeling of two-hot-arm Horizontal Thermal Actuator," J. of Micromechanics and Microengineering, Vol. 13, pp. 312-322, 2003.

[15] Mohammad A. Mayyas, Woo H. Lee, Dan Popa, Panos Shiakolas, Ping Zhang and Harry E. Stephanou. "Comprehensive Electrothermal Modeling of a Thermal Microgripper," 2005 TexMEMS, The VII International Conference on Micro Electro Mechanical Systems, Sept. 21-22, 2005.

[16] Dong Yan, Amir Khajepour, and Raafat Mansour, "Design and modeling of a MEMS bidirectional vertical thermal actuator," Journal of Micromechanics and Microengineering, Vol. 14, pp. 841-850, 2004.

[17] J. Denavit, R. S. Hartenberg, "A Kinematic Notation for Lower-Pair Mechanisms Based on Matrices," Trans. of the ASME J., Appl. Mech., Vol. 77, pp. 215-221, 1955.

[18] Shyam Venugopal, Design of a high accuracy, three degree of freedom MEMS robot for biomedical applications, Thesis, University of Texas at Arlington, 2004.

[19] N. Fazenda, T.-F. Niaritsiry and R. Clavel, "Simulation-based Kinematic Modelling of a High Precision Parallel Manipulator," Proceedings of the 9th IEEE International Conference on Methods and Models in Automation and Robotics, Miedzyzdroje, Poland, 2003. 